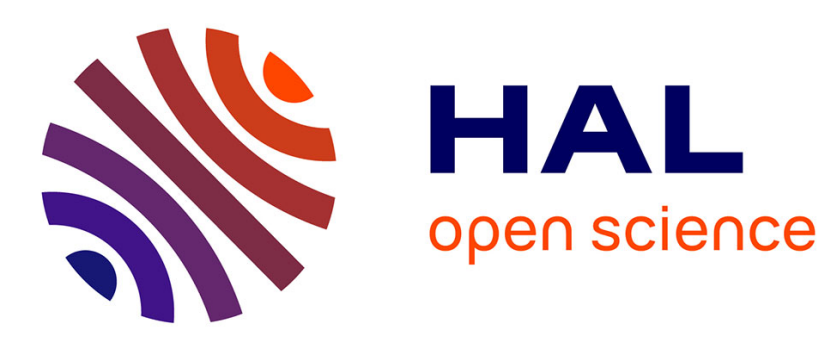

\title{
A posteriori error estimation for stochastic static problems
}

\author{
Hung Mac, Stéphane Clenet
}

\section{To cite this version:}

Hung Mac, Stéphane Clenet. A posteriori error estimation for stochastic static problems. IEEE Transactions on Magnetics, 2014, 50 (2), 10.1109/TMAG.2013.2281103 . hal-01017925

\section{HAL Id: hal-01017925 https://hal.science/hal-01017925}

Submitted on 3 Jul 2014

HAL is a multi-disciplinary open access archive for the deposit and dissemination of scientific research documents, whether they are published or not. The documents may come from teaching and research institutions in France or abroad, or from public or private research centers.
L'archive ouverte pluridisciplinaire HAL, est destinée au dépôt et à la diffusion de documents scientifiques de niveau recherche, publiés ou non, émanant des établissements d'enseignement et de recherche français ou étrangers, des laboratoires publics ou privés. 


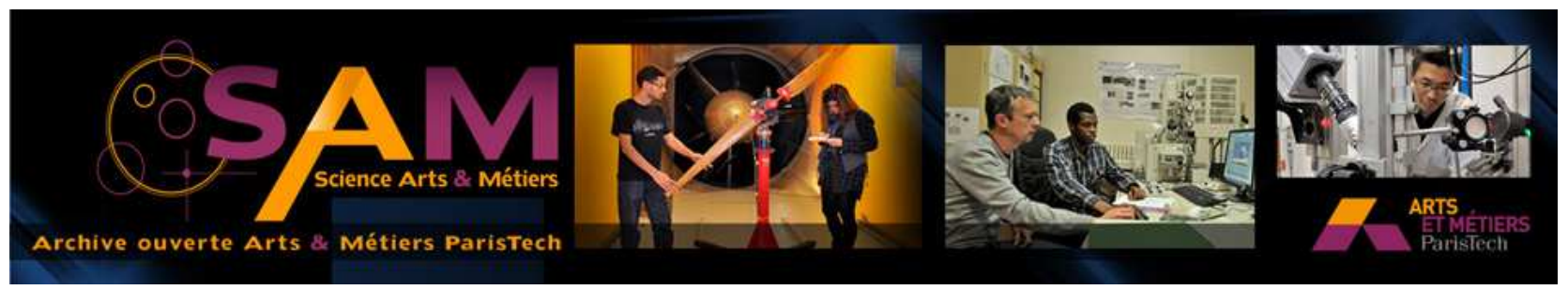

Science Arts \& Métiers (SAM)

is an open access repository that collects the work of Arts et Métiers ParisTech researchers and makes it freely available over the web where possible.

This is an author-deposited version published in: http://sam.ensam.eu Handle ID: .http://hdl.handle.net/10985/8319

\section{To cite this version :}

Hung MAC, Stéphane CLENET - A posteriori error estimation for stochastic static problems IEEE transactions on Magnetics - Vol. 50, n², p.nc - 2014 


\title{
A posteriori error estimation for stochastic static problems
}

\author{
D. H. Mac ${ }^{1}$, S. Clénet ${ }^{1}$ \\ ${ }^{1}$ L2EP, Arts et Métiers ParisTech, 8 bd de Louis XIV, 59000 Lille, France
}

\begin{abstract}
To solve stochastic static field problems, a discretization by the Finite Element Method can be used. A system of equations is obtained with the unknowns (scalar potential at nodes for example) being random variables. To solve this stochastic system, the random variables can be approximated in a finite dimension functional space - a truncated polynomial chaos expansion. The error between the exact solution and the approximated one depends not only on the spatial mesh but also on the discretization along the stochastic dimension. In this paper, we propose an a posteriori estimation of the error due to the discretization along the stochastic dimension.
\end{abstract}

Index Terms-Stochastic problems, Finite Element Method, Polynomial Chaos Expansion, Magnetostatics, Error estimation.

\section{INTRODUCTION}

Numerical modelling can be used to predict the behavior of an electromagnetic device. The Maxwell equations can be solved using the Finite Element Method (FEM). In a linear static problem, FEM leads to a linear system of equations. The unknowns could be the value of the scalar potential at the nodes or the circulation of the vector potential at the edges, for example.

When the input data (behavior laws of the material, the geometry of the device, ...) of the numerical model are uncertain due to several factors like the ageing of the material, the imperfections of the manufacturing processes, ..., the Maxwell equations become stochastic. Consequently, system of equations obtained by FEM becomes also stochastic. The unknowns are random variables. Sampling methods like the Monte Carlo Simulation Method (MCSM) can be used to estimate statistical moments or probability of failures. Another method consists of approximating the solution of the system of equations given by FEM along the random dimension. In engineering, this approach was introduced by Ghanem [1] in mechanics and widely developed in this area. In computational electromagnetic, this approach was introduced in [2] and [3].

The Polynomial Chaos Expansion (PCE) [4] is one of the most popular methods to approximate random variables. The numerical solution can be obtained then by two discretization steps, one along the spatial dimension (mesh) and the other along the random dimension using a truncated PCE. To evaluate the numerical error (i.e. the distance between the numerical solution and the exact solution), one can distinguish two kinds of error estimation, a priori and a posteriori error estimations. In this paper, we are interested in a posteriori error estimation which is calculated from the numerical solution and so, the error estimation is evaluated after the numerical solution of the problem. The a posteriori error estimation of a stochastic problem has been already addressed in literature [5], [6], [7]. In [5], an error estimation based on the hyper-circle theorem is proposed. This error requires the solutions of two complementary formulations. This error estimator takes simultaneously into account the error due to the discretizations along the spatial and the random dimensions, giving a so-called "global estimation". In [6], an error estimation based on the solution of an adjoint problem is proposed. The estimator is applied to some non-linear problems where the adjoint problem becomes linear and the solution of the adjoint problem needs then less time than the initial one. The error estimation in [6] is also global and can be applied only for the numerical solution obtained by the Spectral Stochastic Finite Element Method (SSFEM) [2], [3]. In [7], an error estimator enabling us to evaluate the error due the stochastic discretization (only due to PCE), the so-called stochastic error, has been proposed. This estimator is based on the solution of the error equation where the right hand side is the residual evaluated from the numerical solution. The error equation has to be solved using a PCE with higher order than the one used for the numerical solution of the initial equation. In this method, two stochastic problems have to be solved.

In this paper, we propose a stochastic error estimator that is evaluated directly from the residual calculated from the numerical solution. The proposed error estimation is tested on an academic example.

\section{Magnetostatic PRoblem With UNCERTAinties ON THE BeHaVioR LAW}

We are interested in a magnetostatic problem defined on a domain $D$ with an uncertain permeability. The permeability can be modeled by a random field $\mu(x, \theta)$ where the parameter $\theta$ refers to an elementary event and $x$ the position. We assume that the random field $\mu(x, \theta)$ can be expressed explicitly (or at least approximated by a Karhunen-Loeve expansion [8]) as a function of a random vector $\boldsymbol{\xi}(\theta)=\left(\xi_{1}(\theta), \xi_{2}(\theta), \ldots, \xi_{M}(\theta)\right)$ where $\xi_{1}(\theta), \xi_{2}(\theta), \ldots, \xi_{M}(\theta)$ are real independent random variables with known probability density functions. We denote $\Theta^{M} \subset \mathbf{R}^{M}$ the domain of the random vector $\boldsymbol{\xi}(\theta)$. In the following, to simplify the notations, the dependency on $\theta$ of the random vector $\boldsymbol{\xi}$ will be removed. We assume also that the permeability is bounded, meaning that:

$$
0<\mu_{\min }(x) \leq \mu(x, \boldsymbol{\xi}) \leq \mu_{\max }(x)<\infty \quad \forall \boldsymbol{\xi} \subset \Theta^{M}
$$


The equations of the magnetostatic problem defined on $D$, can be written as:

$$
\left\{\begin{array}{l}
\nabla \cdot \mathbf{B}(x, \xi)=0 \\
\nabla \times \mathbf{H}(x, \xi)=\mathbf{J}_{s}(x) \\
\mathbf{B}(x, \xi)=\mu(x, \xi) \mathbf{H}(x, \xi)
\end{array}\right.
$$

with $x \in D, \xi \in \Theta^{M}$, where $\mathbf{H}$ and $\mathbf{B}$ are the magnetic field and the magnetic flux density, respectively. The source term $\mathbf{J}_{s}$, being divergence free, can be written as $\mathbf{J}_{s}(x)=\nabla \times \mathbf{H}_{s}(x)$. For the sake of simplicity, homogeneous boundary conditions are prescribed:

$$
\mathbf{B}(x, \xi) \cdot \mathbf{n}(x)=0 \quad \forall x \in \Gamma_{D}, \forall \xi \in \Theta^{M} .
$$

We should mention that the results proposed in this paper can be extended also to the case with the mixed boundary conditions, i.e $\mathbf{B}(x, \xi) \cdot \mathbf{n}(x)=0$ on one part of $\Gamma_{D}$ and $\mathbf{H}(x, \xi) \times$ $\mathbf{n}(x)=0$ on the complementary part of the boundary $\Gamma_{D}$. To solve the problem described in (2) and (3), the scalar potential $\Omega$ can be introduced such that:

$$
\mathbf{H}(x, \xi)=-\nabla \Omega(x, \xi)+\mathbf{H}_{s}(x)
$$

or the vector potential $\mathbf{A}$, such that:

$$
\mathbf{B}(x, \xi)=\nabla \times \mathbf{A}(x, \boldsymbol{\xi}) .
$$

In this paper, we will focus on the scalar potential formulation. The extension to the vector potential formulation case is straightforward. The scalar potential formulation yields the following weak form:

$$
\begin{gathered}
\int_{D} \mu(x, \boldsymbol{\xi}) \nabla \Omega(x, \boldsymbol{\xi}) \cdot \nabla \lambda(x) d x= \\
\int_{D} \mu(x, \boldsymbol{\xi}) \mathbf{H}_{s}(x) \cdot \nabla \lambda(x) d x \quad \forall \lambda \in H^{1}(D), \forall \boldsymbol{\xi} \in \Theta^{M}
\end{gathered}
$$

where $H^{1}(D)$ is a functional space defined by:

$$
H^{1}(D)=\left\{\left.v \in L^{2}(D)\left|\int_{D}\right| \nabla v(x)\right|^{2} d x<\infty\right\} .
$$

Clearly, the solution of (4) is defined up to an additive constant. However, one can notice that the magnetic field is unique and does not depend on the additive constant. In a general case, the analytical solution of (4) is not reachable. Thus, we introduce a tetrahedral mesh $\mathcal{M}$ of the domain $D$ with $n_{0}$ nodes, $n_{1}$ edges, $n_{2}$ facets and $n_{3}$ elements. An approximation $\Omega_{h}$ of the scalar potential $\Omega$ is sought in $V_{h}^{i_{0}}$ such that

$$
\begin{gathered}
\int_{D} \mu(x, \boldsymbol{\xi}) \nabla \Omega_{h}(x, \boldsymbol{\xi}) \cdot \nabla w(x) d x= \\
\int_{D} \mu(x, \boldsymbol{\xi}) \mathbf{H}_{s}(x) \cdot \nabla w(x) d x \quad \forall w \in V_{h}^{i_{0}}, \forall \boldsymbol{\xi} \in \Theta^{M}
\end{gathered}
$$

where the discrete functional space $V_{h}^{i_{0}}$ is defined by:

$$
V_{h}^{i_{0}}=\operatorname{span}\left\{w_{0 i} \mid i=1,2, \ldots, i_{0}-1, i_{0}+1, \ldots n_{0}\right\}
$$

with $w_{0 i}$ the shape function [9] associated with the node $i$. One can notice that the choice of the discrete functional space
$V_{h}^{i_{0}}$ imposes a gauge condition such that the scalar potential is equal to zero at the node $i_{0}$. This gauge condition imposes the uniqueness of the solution of (6). The other gauge conditions [13] can be implemented but this has no consequence on our proposed method.

\section{Polynomial chaos expansion}

The solution of (6) can be written under the form

$$
\Omega_{h}(x, \boldsymbol{\xi})=\sum_{i=1, i \neq i_{0}}^{n_{0}} \Omega_{h}^{i}(\boldsymbol{\xi}) w_{0 i}(x)
$$

with $\Omega_{h}^{i}(\boldsymbol{\xi})$ the value of the scalar potential at the node $i$ that is a random variable. To obtain an explicit expression of $\Omega_{h}^{i}(\boldsymbol{\xi})$, the idea is to approximate it in a finite dimension functional space. A truncated PCE can be an appropriate choice if the variation of $\Omega_{h}^{i}(\xi)$ as a function of $\boldsymbol{\xi}$ is quite smooth. The scalar potential $\Omega_{h}(\boldsymbol{\xi})$ at the node $i$ is then approximated by:

$$
\Omega_{h}^{i}(\boldsymbol{\xi}) \approx \sum_{j=0}^{P} \Omega_{i j} \Psi_{j}(\boldsymbol{\xi})
$$

where $\Psi_{j}, j=0: P$ is a set of orthonormal polynomials [4] and $\Omega_{i j}$ are the real coefficients to be determined. The numerical solution of the problem can be written as:

$$
\Omega_{h, P}(x, \boldsymbol{\xi})=\sum_{j=0}^{P} \sum_{i=1, i \neq i_{0}}^{n_{0}} \Omega_{i j} w_{0 i}(x) \Psi_{j}(\boldsymbol{\xi}) .
$$

Two categories of methods, non intrusive and intrusive, have been proposed in the literature to determine $\Omega_{i j}$. In the nonintrusive methods [10], [11], the deterministic FEM model can be directly used to obtain the stochastic solution. Indeed, the coefficients $\Omega_{i j}$ are determined by:

$$
\Omega_{i j}=\sum_{k=1}^{Q} \Omega_{h}^{i}\left(\boldsymbol{\xi}_{k}\right) \Psi_{j}\left(\boldsymbol{\xi}_{k}\right) \omega_{k} .
$$

The evaluation of coefficients $\Omega_{i j}$ in (11) requires $Q$ evaluations $\Omega_{h}^{i}\left(\xi_{k}\right)$, for $k=1: Q$, where $\Omega_{h}^{i}\left(\xi_{k}\right)$ refers to the scalar potential $\Omega_{h}$ at node $i$ at well fitted points $\xi_{k}$. Thus, $Q$ deterministic problems (6) corresponding to $Q$ realizations of the permeability $\mu\left(x, \boldsymbol{\xi}_{k}\right)$ have to be solved. Several choices of the weights $\omega_{k}$ and of the points $\xi_{k}$ are possible (see [10], [11]). For a given mesh, the numerical solution (10) depends on the choice of the set of polynomials $\Psi_{j}$ and on the set of the evaluation points $\boldsymbol{\xi}_{k}$ and on the associated weights $\omega_{k}$.

In the intrusive method (SSFEM method [2], [3]), the coefficients $\Omega_{i j}$ are determined by using the Galerkin projection:

$$
\begin{gathered}
\mathrm{E}\left(\int_{D} \mu(x, \boldsymbol{\xi}) \nabla \Omega_{h, P}(x, \boldsymbol{\xi}) \cdot \nabla w_{0 i}(x) d x \Psi_{j}(\boldsymbol{\xi})\right)= \\
\mathrm{E}\left(\int_{D} \mu(x, \boldsymbol{\xi}) \mathbf{H}_{s}(x) \cdot \nabla w_{0 i}(x) d x \Psi_{j}(\boldsymbol{\xi})\right)
\end{gathered}
$$

with $i=1: n_{0} \backslash i_{0}, j=0: P$ and $\mathrm{E}(X(\xi))$ denoting the expectation of the random variable $X(\xi)$. Equation (12) leads to a linear matrix system of dimension $\left(n_{0}-1\right) \times(P+1)$ where the solution is the vector of the coefficients $\Omega_{i j}$. For a given mesh, the numerical solution (10) depends on the choice of 
the set of polynomials and on the accuracy of the solution of the linear system given by (12).

\section{Stochastic ERror EStimation}

\section{A. Definition of the numerical errors}

The stochastic error $e_{s t o}$ is defined by the following

$$
\begin{aligned}
e_{s t o}^{2}(\xi)= & \int_{D} \mu(x, \boldsymbol{\xi}) \nabla\left(\Omega_{h, P}(x, \boldsymbol{\xi})-\Omega_{h}(x, \boldsymbol{\xi})\right) . \\
& \nabla\left(\Omega_{h, P}(X, \boldsymbol{\xi})-\Omega_{h}(x, \boldsymbol{\xi})\right) d x .
\end{aligned}
$$

Using the same norm, the spatial error $e_{s p a}$ and the global error $e_{g l o}$ can also be defined respectively as the distance between $\Omega_{h}$ and $\Omega$ and the distance between $\Omega_{h, P}$ and $\Omega$. From (4), (6) and (10) it can be shown that

$$
e_{g l o}^{2}(\xi)=e_{s t o}^{2}(\xi)+e_{s p a}^{2}(\xi) .
$$

The global error estimation requires estimations of both the stochastic error $e_{s t o}$ and the spatial error $e_{s p a}$. In the following, we will focus only on the estimation of the stochastic error $e_{\text {sto }}$ which is directly linked to the discretization of $\Omega_{h}$ along the stochastic dimension.

\section{B. Stochastic error estimator}

We propose the following estimator for $e_{s t o}^{2}(\xi)$

$$
\eta_{s t o}^{2}(\boldsymbol{\xi})=\mathbf{R}^{t}(\boldsymbol{\xi}) \boldsymbol{\Lambda}_{0}^{-1} \mathbf{R}(\boldsymbol{\xi})
$$

where $\mathbf{R}$ is a stochastic residual vector whose coefficients are given by:

$$
\begin{gathered}
{[\mathbf{R}]_{i}(\boldsymbol{\xi})=\int_{D} \mu(x, \boldsymbol{\xi}) \nabla \Omega_{h, P}(x, \boldsymbol{\xi}) \cdot \nabla w_{0 i}(x) d x-} \\
\int_{D} \mu(x, \boldsymbol{\xi}) \nabla \mathbf{H}_{s}(x) \cdot \nabla w_{0 i}(x) d x
\end{gathered}
$$

with $i=1: n_{0} \backslash i_{0}$ and $\boldsymbol{\Lambda}_{0}$ the expectation of the stiffness matrix defined by:

$$
\left[\boldsymbol{\Lambda}_{0}\right]_{i j}=\int_{D} \mathrm{E}(\mu(x, \boldsymbol{\xi})) \nabla w_{0 i}(x) \cdot \nabla w_{0 j}(x) d x
$$

with $i=1: n_{0} \backslash i_{0}, j=1: n_{0} \backslash i_{0}$. It can be shown that $\eta_{\text {sto }}$ in (15) is an equivalent measure of the stochastic error $e_{s t o}$ meaning that

$$
k_{1} \eta_{s t o}^{2}(\boldsymbol{\xi}) \leq e_{s t o}^{2}(\boldsymbol{\xi}) \leq k_{2} \eta_{s t o}^{2}(\boldsymbol{\xi})
$$

with $k_{1}$ and $k_{2}$ are positive coefficients and independent of $\Omega_{h}$ and of $\Omega_{h, P}$. In particular, we can deduce an explicit expression of these coefficients:

$$
\begin{aligned}
& k_{1}=\min _{x \in D}\left(\frac{\mathrm{E}(\mu(x, \boldsymbol{\xi}))}{\mu_{\max }(x)}\right) \\
& k_{2}=\max _{x \in D}\left(\frac{\mathrm{E}(\mu(x, \boldsymbol{\xi}))}{\mu_{\min }(x)}\right)
\end{aligned}
$$

(see the APPENDIX for the proof of (18)). Due to the fact that $\eta_{\text {sto }}^{2}(\xi) \geq 0, \forall \xi \subset \Theta^{M}$, and from (18), the expectation $\mathrm{E}\left(\eta_{s t o}^{2}(\xi)\right)$ could be an appropriate indicator to quantify the stochastic error $e_{\text {sto }}$. To evaluate $\mathrm{E}\left(\eta_{\text {sto }}^{2}(\xi)\right)$, the residual $\mathbf{R}$ is deduced from (16) as shown:

$$
\mathbf{R}(\xi)=\sum_{j=0}^{P_{1}} \mathbf{R}_{j} \Psi_{j}(\xi)
$$

where $\mathbf{R}_{j}$ are real vectors. Due to the fact that $\Psi_{j}(\boldsymbol{\xi})$ are orthonormal, we obtain:

$$
\mathrm{E}\left(\eta_{s t o}^{2}(\xi)\right)=\sum_{j=0}^{P_{1}} \mathbf{R}_{j}^{t} \boldsymbol{\Lambda}_{0}^{-1} \mathbf{R}_{j} .
$$

From (19), the ratio between the upper and the lower bounds of the stochastic error (18) can be evaluated. In practice, this ratio corresponds to a small number of units. Furthermore, the estimation (18) is independent of the method used to solve the stochastic problem (SSFEM, Non intrusive, etc.) and can be extended to the different stochastic approximation bases (truncated PCE in this paper or wavelet decomposition [12], etc.).

\section{Numerical EXAMPLe}

We are interested in a magnetostatic problem presented in Fig. 1. The domain is divided into 5 sub-domains with the

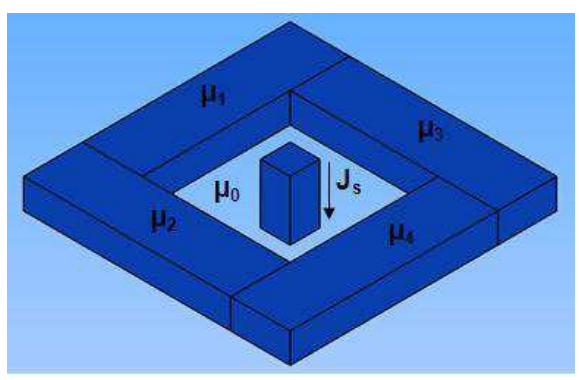

Figure 1: Magnetostatic problem defined in the domain $D$.

relative permeabilities $\mu_{0}=1, \mu_{1}=\mu_{2}=1000 . \mu_{3}$ and $\mu_{4}$ are two independent uniform random variables defined on [600 - 1400]. The current $\mathbf{J}_{s}$ is imposed equal to 1A. We apply the SSFEM method [2] to solve this problem. For a given numerical solution $\Omega_{h, P}(x, \xi)$, the mean value of the stochastic estimator $\eta_{\text {sto }}^{2}$ is compared to the mean value of the stochastic error $e_{s t o}^{2}$ estimated by the MCSM described on the flow chart in Fig. 2. As mentioned in Section III, with a given mesh, the

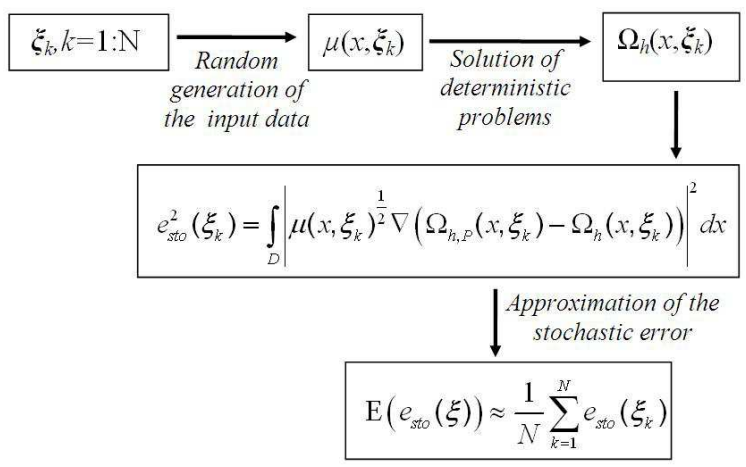

Figure 2: Monte-Carlo method. 
numerical solution (10) depends on the choice of the set of polynomials and on the accuracy of the solution of the SSFEM system of equations given by (12). In this example, a mesh with 2617 nodes and the full tensorised Legendre PCE [4] are used. The set of the polynomials depends then on the order of truncation $p$. The SSFEM linear system of equations is solved using an iterative method and the accuracy of the solution is fixed by a stopping criterion $\varepsilon$ based on a residual norm.

The evolution of the mean value of the stochastic estimator and the mean value of stochastic error estimated by MCSM as a function of the order $p$ of PCE and of the stopping criterion $\varepsilon$ is presented in Fig. 3 .

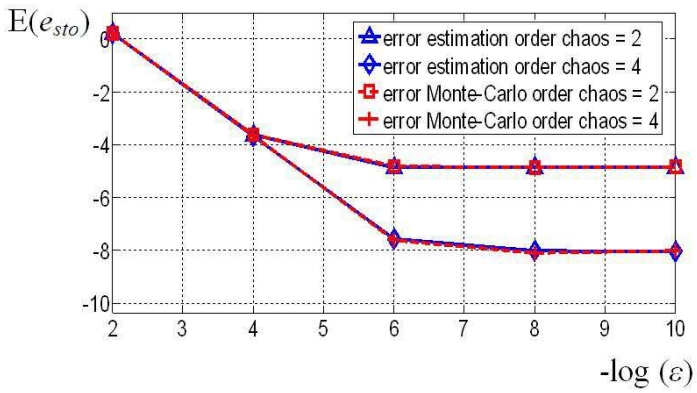

Figure 3: Evolution of the mean value of the estimator and of the MCSM estimated stochastic error.

According to Fig. 3, we can deduce that : -The estimator and the stochastic error estimated by the MSCM are very close. -While the accuracy level of the solution of the SSFEM matrix system is low ( $\varepsilon$ is greater than $10^{-4}$ in Fig. 3) the stochastic error is the same with different orders of PCE. While the accuracy level is quite high $\left(\varepsilon\right.$ is lower than $10^{-4}$ in Fig. 3) a higher order of PCE yields a smaller stochastic error. -With a given order of PCE, when the accuracy level increases, the evolution of the stochastic error decreases up to a given value before being stable $(\log (\varepsilon)=-6$ with order $p=2$ and $\log (\varepsilon)=-8$ with order $p=4)$. It is thus wasteful to increase the accuracy level of the solution of the SSFEM matrix system beyond these points.

\section{ConcLusion}

We have presented a stochastic a posteriori error estimator for a stochastic magetostatic problem. The estimator allows us to evaluate the error due to the stochastic discretization (truncated PCE in this paper). The estimator is based on a residual of the discrete weak formulation. For a given mesh, by using the estimator, one can compare the accuracy of different numerical solutions. In this paper, we are interested only in the stochastic error. In order to obtain a complete analysis, the error due to the spatial discretization has to be taken into account.

\section{REFERENCES}

[1] R. Ghanem, P. Spanos. "Stochastic finite elements: a spectral approach", Springer-Verlag, Berlin, 1991.

[2] R. Gaignaire, S. Clenet, O. Moreau, and B. Sudret. "3D spectral stochastic finite element method in electromagnetism", IEEE Transactions on Magnetics, vol.43, no.4, pp. 1209-1212, 2007
[3] K. Beddek, Y. Le Menach, S. Clénet, O. Moreau. "3D spectral finite element in static electromagnetism using vector potential formulation", IEEE Transactions on Magnetics, vol.47, pp. 1250-1253, 2011.

[4] D. Xiu, G.E. Karniadakis. "The Wiener-Askey polynomial chaos for stochastic differential equations", SIAM J. Sci. Comput, vol. 24, no. 2, pp. 619-644, 2002.

[5] S. Clénet, N. Ida. "Error Estimation in a Stochastic Finite Element Method in electrokinetics", Int. J. Numer. Meth. Eng., vol. 81, no. 11, pp. 1417$1438,2010$.

[6] O. Le Maitre, O. Knio, H. Najm and R. Ghanem. "Adaptive multiwavelets decomposition for stochastic processes", ICOSAHOM, Brown University, Providence RI, June 21-25, 2004.

[7] X. Wanand, G. E. Karniadakis. "Error Control in Multi-Element Generalized Polynomial Chaos Method for Elliptic Problems with Random Coefficients", Communications in Computational Physics, vol. 5, no. 2-4, pp. 793-820, 2009

[8] S.P. Huang, S.T. Quek and K.K. Phoon. "Convergence study of the truncated Karhunen - Loeve expansion for simulation of stochastic processes", Int. J. Numer. Meth. Eng., vol. 52, issue 9, pp. 1029 - 1043 , 2001.

[9] A. Bossavit. "Computational electromagnetism", Academic Press (Boston), 1998.

[10] M. Berveiller, B. Sudret et M. Lemaire. "Stochastic finite element: a non intrusive approach by regression”, European Journal of Computational Mechanics, vol. 15, no. 1-2-3, pp. 81-92, 2006.

[11] K. Beddek, S. Clénet, O. Moreau, V. Costan, Y. Le Menach, A. Benabou. "Adaptive Method for Non-Intrusive Spectral Projection, Application on a Stochastic Eddy Current NDT Problem", IEEE Transactions on Magnetics, vol. 48, no. 2, pages. 759-762, 2012

[12] O. Le Maitre, O. Knio, H. Najm and R. Ghanem. "Adaptive multiwavelets decomposition for stochastic processes", ICOSAHOM, Brown University, Providence RI, June 21-25, 2004.

[13] L. Kettunen, K. Forsman, A. Bossavit. "Gauging in Whitney spaces ", IEEE Transactions on Magnetics, vol. 35, Issue: 3, pp. 1466 - 1469, 1999.

\section{APPENDIX}

(18) can be proved by using the two following lemmas. Lemma 1. For $\Lambda(\xi)$ the stiffness matrix defined by:

$$
[\mathbf{\Lambda}]_{i j}(\boldsymbol{\xi})=\int_{D} \mu(x, \boldsymbol{\xi}) \nabla w_{0 i}(x) \cdot \nabla w_{0 j}(x) d x
$$

with $i=1: n_{0} \backslash i_{0}, j=1: n_{0} \backslash i_{0}$, we have

$$
e_{s t o}^{2}(\xi)=\mathbf{R}^{t}(\xi) \Lambda^{-1}(\xi) \mathbf{R}(\xi)
$$

Lemma 2. For $\Lambda_{1}(\xi)$ and $\Lambda_{2}(\xi)$, two matrices of dimension $\left(n_{0}-1\right) \times\left(n_{0}-1\right)$ such that:

$$
\begin{aligned}
& {\left[\boldsymbol{\Lambda}_{1}\right]_{i j}(\boldsymbol{\xi})=\int_{D} \mu_{1}(x, \boldsymbol{\xi}) \nabla w_{0 i}(x) \cdot \nabla w_{0 j}(x) d x} \\
& {\left[\boldsymbol{\Lambda}_{2}\right]_{i j}(\boldsymbol{\xi})=\int_{D} \mu_{2}(x, \boldsymbol{\xi}) \nabla w_{0 i}(x) \cdot \nabla w_{0 j}(x) d x}
\end{aligned}
$$

with $0<\mu_{1}(x, \boldsymbol{\xi}) \leq \mu_{2}(x, \boldsymbol{\xi}), \forall x \in D, \forall \boldsymbol{\xi} \in \Theta^{M}$. We thus obtain:

$$
\mathbf{R}^{t}(\boldsymbol{\xi}) \boldsymbol{\Lambda}_{2}^{-1}(\boldsymbol{\xi}) \mathbf{R}(\boldsymbol{\xi}) \leq \mathbf{R}^{t}(\xi) \boldsymbol{\Lambda}_{1}^{-1}(\xi) \mathbf{R}(\xi), \forall \boldsymbol{\xi} \in \Theta^{M}
$$

From lemma 1 and lemma 2, one can deduce (18) due to the fact that

$$
\frac{1}{k_{2}} \mathrm{E}(\mu(x, \boldsymbol{\xi})) \leq \mu(x, \boldsymbol{\xi}) \leq \frac{1}{k_{1}} \mathrm{E}(\mu(x, \boldsymbol{\xi}))
$$

$\forall x \in D, \forall \xi \in \Theta^{M}$.

\section{ACKNOWLEDGEMENTS}

This work is supported by the program MEDEE funded by the Nord Pas de Calais council and the European Community. 